Recepción: 28/12/2018

Aceptación: 22/12/2018

Publicación: 05/03/2019

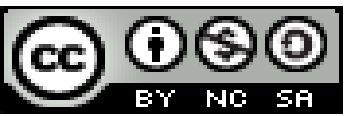

Ciencias de la Salud

Artículo de investigación

\title{
Planes terapéuticos para el abordaje de problemáticas infanto-juveniles en contextos familiares
}

Therapeutic plans for the treatment of child-juvenile problems in family contexts

\section{Planos terapêuticos para a abordagem de problemas infanto-juvenis em contextos familiares}

\author{
Gabriela Estefanía Román-Celi ${ }^{\mathrm{I}}$ \\ gabbyrc15@hotmail.es
}

María Magdalena Espinoza-Herrera II lamagddy_20@hotmail.com

Michelle Ivanova Aldeán-Riofrío III mivanova_111@hotmail.com

Correspondencia: gabbyrc15@hotmail.es

\footnotetext{
I Magíster en Pedagogía, Licenciada en Ciencias de la Educación en la Especialidad de Psicología Infantil y Educación Parvularia, Docente de la Universidad Nacional de Loja, Loja, Ecuador.

II Magíster en Terapia Familiar, Licenciada en Psicorehabilitación y Educación Especial, Docente de la Universidad Nacional de Loja, Loja, Ecuador.

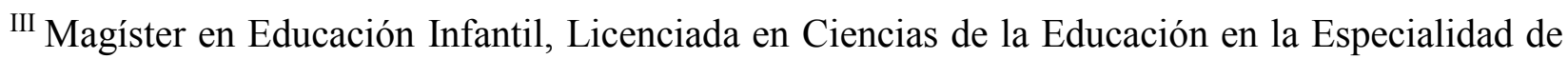
Psicología Infantil y Educación Parvularia, Profesora en Ciencias de la Educación en la Especialidad de Psicología Infantil y Educación Parvularia, Docente de la Universidad Nacional de Loja, Loja, Ecuador.
} 


\section{Resumen}

El presente artículo se propuso explorar las etapas de un plan terapéutico sistemático, en familia con problemas infanto-juvenil del Cantón Loja. La metodología fue de tipo descriptiva transversal, con un diseño no experimental. La población la constituyeron 10 familias que habitan en el Cantón Loja, que previamente fueron evaluadas con problemas infanto-juveniles en su entorno familiar. La recolección de la información se realizó mediante la técnica de la entrevista con un instrumento semi- estructurado y validado a través del juicio de expertos. El análisis estadístico de los datos se llevó a cabo mediante la estadística descriptiva. Los resultados, en mayor porcentajes, indicaron que el $87 \%$ de los investigados consideraron que se debe dar en la etapa de apertura, una secuencia de los hechos que acompañan la vida del niño y/o adolescente; el $94 \%$ considero que se han de utilizar técnicas muy variadas y de muchas fuentes para dar seguimiento al plan terapéutico familiar y el $70 \%$ indico de forma positiva que el cierre de un plan terapéutico se debe dar luego de hacer una evaluación o un seguimiento para el inicio de la apertura al plan terapéutico familiar. Entre sus conclusiones se indicó un cumplimiento de forma afirmativa de las etapas o fases del plan terapéutico entre las familias investigadas.

Palabras clave: planes terapéuticos; problemáticas infanto-juveniles; sistema terapéutico y familia

\section{Abstract}

This article is proposed to explore the stages of a systematic treatment plan in family with adolescent problems of the Canton of Loja. The methodology was transverse descriptive, with a non-experimental design. Constituted the population 10 families who live in the Canton of Loja, which previously were evaluated with infanto-juvenile problems in their family environment. The data collection was made using the technique of the interview with an instrument of semi structured and validated through expert opinion. The statistical analysis of data was carried out using descriptive statistics. The results, in greater percentages indicated that $87 \%$ of those investigated were considered that it should be in the opening stage, a sequence of events that accompany the life of the child or adolescent; $94 \%$ consider that very varied techniques should be used and from many sources to track the family therapeutic plan and $70 \%$ Indian positively that the closure of a therapeutic plan should be given after making an assessment or follow-up to ape 
rtura to family treatment plan. Among their conclusions indicated affirmative way compliance with the stages or phases of the therapeutic plan among the investigated families.

Keywords: therapeutic plans; issues infanto-juvenile; therapeutic system and family

\section{Resumo}

O presente artigo propõe-se a explorar as etapas do plano terapêutico sistémico, em familias com problemas infanto-juvenis do Cantão Loja. La metodología fue de tipo descritiva transversal, com um propósito não experimental. La población la constituyeron 10 familias that habitan en el Cantón Loja, que forneceu avaliações com problemas infanto-juvenis em su entorno familiar. A recolección de la información se realiza através da técnica da entrevista com um instrumento semi-estructurado e validado através do juicio de peritos. A análise estatística dos dados é feita a partir do código da estatística descritiva. Os resultados, em porcentajes do mayor, indicando que os investigadores em investigar que você deve ter começado a fase de abertura, em uma seção dos jogos que acompanham a vida do bebê / adolescente; el 94\% considere que o plano terapêutico seja bem variado e adequado para o planejamento terapêutico familiar $70 \%$ ideal de forma que o plano terapêutico seja debatido de acordo com a avaliação de um seguimiento parágrafo o inicio da abertura do plano terapêutico familiar. As conclusões deste inquérito são indicativas de acumulação de forma afirmativa das fases do plano terapêutico terapêutico entre as familias investigadas.

Palabras clave: planos terapêuticos; problemáticas infanto-juvenis; sistema terapêutéutico y familia

\section{Introducción}

La familia es el primer contexto, en la que están presentes tanto las prácticas de disciplina familiar, el tipo e intensidad del vínculo afectivo, el contacto físico y la predictibilidad del contexto, y como consecuencia de todo ello, el apego familiar. De allí, que se ha considerado que la identidad de sus miembros, según Gracia y Musitu (2000), se desarrolla dentro de un contexto, donde se dan procesos a través del clima familiar, los tipos de socialización que los padres utilizan y los grados y modos de comunicación entre los padres y sus hijos.

Antes situaciones previstas del contexto familiar, surgen los planes terapéuticos familiares como un proceso de acompañamiento a las personas en sus dificultades para encontrar alternativas a las 
mismas. Sin embargo, la psicoterapia en general, tiene raíces que van más allá de lo que se puede ver hoy y en este recorrido se pueden encontrar no solo variados sistemas conceptuales que hoy viven y conviven en el quehacer de los psicoterapeutas, sino que también se debe recordar que estos sistemas conceptuales se refieren o tienen relación con el contexto en el cual se han desarrollado.

La manifestación de nuevas problemáticas infanto - juveniles, obliga a los profesionales que quieren acompañar a las familias a presentar alternativas a sus dificultades, a localizar otras formas de trabajar con ellas y de posicionarse, para poder realizar su acompañamiento. Con base en lo expuesto se exploraron las tres etapas de un plan terapéutico sistemático en familia con problemas infanto-juvenil del Cantón de Loja.

\section{Desarrollo}

Todo proceso terapéutico pasa por varias fases; todas ellas son muy importantes en distintos niveles. Según Gimeno (1999), se pueden distinguir tres fases; la apertura o formación del Sistema Terapéutico, el Proceso Terapéutico en sí mismo y la evaluación y cierre. Una de las principales razones, plantea Garaigordobil y col (2005), para escoger estos planes terapéuticos es que la práctica clínica enseña que, en líneas generales, existen estos tres momentos en el contacto con los consultantes, aunque, por supuesto, en la práctica cotidiana no existe una clara delimitación entre uno y otro, así como tampoco existe un límite entre los elementos considerados al interior de cada una de las fases.

La razón fundamental para plantear las tres fases, es que existe un isomorfismo entre el proceso terapéutico en su totalidad y en cada una de las entrevistas realizadas, ya que éstas también se abren, se desarrollan y se cierran. Este isomorfismo, además, es bastante vital, puesto que la terapia en contextos familiares se da de forma sistemática.

\section{Etapa de apertura o de formación del sistema terapéutico}

En esta etapa se consideran desde el origen de formación en los primeros años de vida del niño, así como de igual forma, mucho de lo que sucederá en un proceso terapéutico se juega en los primeros minutos de una consulta. También, se considera la forma en que el niño y el adolescente entran en relación con los otros, su capacidad de dar confianza, la contención y acogida que el mundo les ofrece y la forma en que se posicionan y adaptan a lo que les ofrece su alrededor, es de 
señalar todas aquellas aperturas que se les ofrece e indican las posibilidades, pero que también marcan los límites, determinan los contextos y que influyen en sus relaciones. En esta etapa de apertura, se da una secuencia de los hechos, ya que se marca un momento como inicial, cuando en la lógica temporal, este momento forma parte de otros momentos más, puesto que la formación del sistema terapéutico forma parte de otro proceso más amplio que lo contiene.

Una familia que aborda los problemas infanto-juveniles a partir de planes terapéuticos, a menudo consideran algunos momentos, según Gimeno (1999) tales como; la llamada telefónica, el contexto de intervención, el encuadre, el análisis de la demanda, la definición de la relación, el contrato terapéutico y la relación terapéutica.

La llamada telefónica a menudo, refiere al primer contacto que se hace a través de una llamada telefónica, en la que este primer encuentro puede verse como una "cita a ciegas", así marcado el inicio de la relación, se pueden experimentar muchas emociones en este primer encuentro: ansiedad, temor, expectativa e incertidumbre, sin saber muy bien lo que va a suceder posterior al planteamiento de las problemáticas enfrentadas por la familia.

El contexto de intervención, refiere durante esta etapa el momento de las reflexiones que permiten conocer el contexto en el cual se va a intervenir, este momento es importante para la terapia ya que en ella se definen las interacciones que se mantengan en su interior, la cuales se adaptan a las condiciones establecidas por el contexto. En este sentido, Bateson (1999) expresa que, si no se identifica el contexto, no puede comprenderse nada.

El Encuadre, permite durante el proceso de aplicación de un plan terapéutico delimitar el espacio y determinar el tipo de contexto en donde el terapeuta y los consultantes van a desenvolverse. En este sentido Edmond Gilliéron (s/f), indica que el marco terapéutico es el conjunto de factores que comprenden desde las determinantes socioculturales del tratamiento hasta ciertos parámetros más o menos fijos como el lugar, la frecuencia y la duración de las consultas.

El análisis de la demanda, en este momento se elabora en el primer encuentro con un individuo o una familia, cualquiera que sea el contexto y se enfatiza en ello puesto que es clave para el cumplimiento del plan terapéutico, para así evitar que, pese a nuestra buena voluntad y 
conocimientos, vayamos demasiado rápido en el cumplimiento de las tareas a realizar para el cumplimiento de los planes terapéuticos propuestos.

La definición de la relación, según Guang (2003), describe que, durante el intercambio, en la que intervienen una o varias personas, a más de los mensajes concernientes a los contenidos, también pasan otros mensajes que tiene que ver con la definición de la relación. Toda comunicación y, por lo mismo, toda interacción implica inevitablemente la definición de la relación entre los participantes. Se trata de una noción compleja, frontera que separa el espacio terapéutico del espacio social y delimita una zona privilegiada, donde los actos que se realizan y las palabras que se intercambian tienen un valor terapéutico.

El contrato terapéutico implica explicitar, de la manera más clara posible, las condiciones en las cuales se realizará el proceso terapéutico. Este contrato incluye el número de sesiones, lo que implica determinar si existe un número específico de encuentros o se espera hasta ver cómo avanza el proceso.

La relación terapéutica, en este momento del cumplimiento de la primera fase, permite establecer una alianza con el consultante, a través de un compromiso mutuo. Para Gérard Salem (1987), la define como la manera en la cual se desarrolla la relación y que depende en primer lugar de la personalidad del terapeuta, cada uno tiene su manera de comprometerse y de entrar en la relación con sus consultantes.

\section{Etapa de intermedio: la segunda fase o el proceso terapéutico}

En esta etapa del proceso, ya se pueden hacer algunas intervenciones que resulten terapéuticas en los primeros momentos del encuentro con la familia. La división en fases es arbitraria y didáctica, ya que cuando se entra en la relación terapéutica no se puede decir "he terminado el análisis de la demanda, ahora tengo que redefinir la relación". Durante esta etapa, la tarea de comprender de mejor manera lo que sucede en la familia y para esto ayuda el tema de la evaluación y diagnóstico familiar. Se pueden mencionar como elementos que intervienen en esta etapa de evaluación y diagnostico familiar, la estructura y funcionamiento de un sistema, la evolución y los modelos de evaluación familiar.

Por otro lado, es importante indicar que se debe considerar la formulación de hipótesis, las cuales se construyen a partir de la intersección entre lo que la familia lleva a la consulta y, la síntesis que 
el terapeuta hace de su formación teórica, su experiencia profesional y su historia. Asimismo, según Navarro (2006), las técnicas a utilizar han de ser muy variadas y de muchas fuentes, pues son de gran utilidad, tanto en procesos terapéuticos, como de intervención sistémica, formación y supervisión.

Técnica y relación van de la mano, se nutren la una de la otra para facilitar un proceso con la familia, con el infante y/o adolescente. Cabe destacar, que las técnicas se inscriben dentro de un contexto y aparecen en una relación; no son recetas que se aplican sin más; requieren sensibilidad para reconocer el mejor momento de aplicarlas, creatividad para realizarlas y mucha libertad para jugar con ellas.

\section{Etapa final: la tercera fase o el cierre}

En esta etapa, se han de considerar algunas formas de cerrar los procesos terapéuticos, los cuales pueden ser por deserción, de evaluación y cierre, de seguimiento y cierre y a modo de cierre. En cuanto a la etapa final, esta se da luego de hacer una evaluación o también de un seguimiento, lo cual, conduce al sistema terapéutico de nuevo hacia el proceso. El cierre por deserción, en la que la mayoría de los casos terminan en una deserción, pues simplemente el grupo familiar deja de cumplir o ir a las terapias, lo que se convierte en una situación angustiante, sobre todo al inicio de la práctica terapéutica, por desconocer cuales son las causas del abandono de las mismas. La evaluación y cierre, se da cuando ya ha transcurrido un tiempo prudencial o algunas sesiones, también cuando se percibe en el núcleo familiar que las relaciones han cambiado en el sentido que anhelaban y que pueden seguir adelante sin acompañamiento.

El seguimiento y cierre finalmente, para Guay y col (2003), está dado cuando se ha cerrado el proceso con la familia y esta decide solicitar una terapia para una reunión de seguimiento, para verificar que los cambios perduran a través del tiempo. Para este momento, se puede dar el caso en la cual la familia se presente mencionando que, durante el tiempo transcurrido han sucedido muchas cosas, sobre las cuales quisieran un acompañamiento terapéutico para enfrentarlas. Frente a esta nueva demanda, si bien es cierto, se vuelve a un nuevo proceso terapéutico, la familia sale más rápido, debido a que sus miembros han encontrado nuevas formas de enfrentar las circunstancias que están viviendo, por lo cual, el acompañamiento es mucho más corto. 


\section{Metodología}

Se realizó una investigación descriptiva transversal, con un diseño no experimental cuya variable a evaluar fue los planes terapéuticos sistemáticos. La población la constituyeron 10 familias que habitan en el cantón Loja, que previamente fueron evaluadas con problemas infanto-juveniles en su entorno familiar. La recolección de los datos se realizó mediante la técnica de la entrevista con un instrumento semi- estructurado diseñado por los investigadores bajo la forma de cuestionario, el cual fue sometido a juicio de expertos para su validación en la materia. Dicho instrumento estuvo integrado por 9 ítems con preguntas cerradas de alternativas dicotómicas, diseñadas de acuerdo al objeto de estudio y las variables. El análisis estadístico de los datos se realizó mediante la estadística descriptiva como son frecuencias absolutas y relativas, representándolos a través de tablas.

\section{Resultados}

Posterior al análisis de la información obtenida a través de una entrevista realizada a 10 familias residentes del cantón Loja, con problemas infanto-juveniles, en atención al desarrollo de planes terapéuticos sistemáticos de tipo familiar, los mismos se presentan en tablas con sus respectivos análisis.

\section{Tabla1.}

\section{Análisis frecuencial y descriptivo de la etapa de apertura de un plan terapéutico}

\begin{tabular}{c|l|c|c|c}
\hline \multirow{2}{*}{$\mathrm{N}^{\circ}$} & \multicolumn{1}{c|}{ Ítems } & \multicolumn{3}{c}{ Respuestas \% } \\
\cline { 3 - 5 } & \multicolumn{1}{|c|}{$\begin{array}{c}\text { So } \\
\text { ¿Es importante dar a conocer información de los } \\
\text { primeros años de vida del niño y/o adolescente para } \\
\text { dar apertura al plan terapéutico familiar? }\end{array}$} & 75 & 25 & 100 \\
\hline 2 & $\begin{array}{l}\text { ¿Siempre toma en cuenta la forma de posicionarse y } \\
\text { adaptarse del niño y/o adolescente para dar apertura al } \\
\text { plan terapéutico familiar? }\end{array}$ & 68 & 32 & 100 \\
\hline 3 & $\begin{array}{l}\text { iSe debe dar en la etapa de apertura, una secuencia de } \\
\text { los hechos que acompañan la vida del niño y/o } \\
\text { adolescente para dar apertura al plan terapéutico } \\
\text { familiar? }\end{array}$ & 87 & 13 & 100 \\
\hline
\end{tabular}

Fuente: Elaboración propia

En tabla1, se presenta el análisis frecuencial y descriptivo de la etapa de apertura de un plan terapéutico. En la misma se indica que el $75 \%$ de las opiniones si dan importancia a la información de los primeros años de vida del niño y/o adolescente; el 68\% consideraron que 
siempre se toma en cuenta la forma de posicionarse y adaptarse y el $87 \%$ señalaron que se debe dar una secuencia de los hechos que acompañan vida del niño y/o adolescente para dar apertura al plan terapéutico familiar.

\section{Tabla 2.}

\section{Análisis frecuencial y descriptivo de la etapa de intermedio}

\begin{tabular}{|c|c|c|c|c|}
\hline \multirow{2}{*}{$\mathrm{N}^{\circ}$} & \multirow{2}{*}{ Ítems } & \multicolumn{3}{|c|}{ Respuestas \% } \\
\hline & & $\mathrm{Si}$ & No & Total \\
\hline 4 & $\begin{array}{l}\text { ¿Crees que se debe realizar una evaluación y } \\
\text { diagnóstico para dar seguimiento a la apertura de los } \\
\text { planes terapéutico familiar? }\end{array}$ & 81 & 19 & 100 \\
\hline 5 & $\begin{array}{l}\text { ¿Contribuye la formulación de hipótesis, construidas a } \\
\text { partir de la intersección entre lo que la familia lleva a } \\
\text { la consulta y la síntesis que el terapeuta hace de su } \\
\text { formación teórica del niño y/o adolescente para dar } \\
\text { seguimiento al plan terapéutico familiar? }\end{array}$ & 72 & 18 & 100 \\
\hline 6 & $\begin{array}{l}\text { ¿Se deben utilizar técnicas muy variadas y de muchas } \\
\text { fuentes en los niños y/o adolescente para dar } \\
\text { seguimiento al plan terapéutico familiar? }\end{array}$ & 94 & 6 & 100 \\
\hline
\end{tabular}

Fuente: Elaboración propia

En tabla 2, se presenta el análisis frecuencial y descriptivo de la etapa de intermedio de un plan terapéutico. El 81\% de los investigados, coincidieron en opinar de forma afirmativa en que se debe realizar una evaluación y diagnóstico y el $72 \%$ piensa que la formulación de hipótesis contribuye a la consulta y la síntesis que el terapeuta hace de su formación teórica del niño y/o adolescente para dar seguimiento al plan terapéutico familiar. Asimismo, el 94\% considera que deben utilizar técnicas muy variadas y de muchas fuentes en los niños y/o adolescente durante esta etapa terapéutica familiar

\section{Tabla 3.}

Análisis frecuencial y descriptivo de la etapa final o etapa del cierre

\begin{tabular}{c|l|c|c|c}
\hline \multirow{2}{*}{$\mathrm{N}^{\circ}$} & \multicolumn{1}{|c|}{ Ítems } & \multicolumn{3}{c}{ Respuestas \% } \\
\cline { 3 - 5 } & \multicolumn{1}{|c|}{$\mathrm{Si}$} & $\mathrm{No}$ & Total \\
\hline 7 & $\begin{array}{l}\text { ¿Crees que el cierre de un plan terapéutico se debe dar } \\
\text { luego de hacer una evaluación o un seguimiento? }\end{array}$ & 70 & 30 & 100 \\
\hline 8 & $\begin{array}{l}\text { ¿Ha considerado dejar de cumplir o seguir el plan } \\
\text { terapéutico familiar? }\end{array}$ & 36 & 64 & 100 \\
\hline
\end{tabular}




\begin{tabular}{l|l|l|l|l}
9 & $\begin{array}{l}\text { ¿Una vez culminada la terapia familiar, consideras que } \\
\text { puede ser necesario solicitarla de nuevo para una } \\
\text { reunión de seguimiento? }\end{array}$ & 65 & 35 & 100 \\
\hline
\end{tabular}

Fuente: Elaboración propia

En tabla 3, se presenta el análisis frecuencial y descriptivo de la etapa final o etapa del cierre. Se indica que el 70\% de los investigados consideraron de forma positiva que el cierre de un plan terapéutico se debe dar luego de hacer una evaluación o un seguimiento. Por otro lado, el 36\% índico que en algún momento han considerado dejar el plan terapéutico previsto y el $65 \%$ respondió afirmativamente para que una vez culminada la terapia familiar, estiman necesario solicitarla de nuevo para una reunión de seguimiento terapéutico familiar.

\section{Conclusiones}

Existe un alto porcentaje de aceptación de la etapa de apertura de un plan terapéutico, específicamente en relación a la importancia que se le debe dar a la información de los primeros años de vida del niño y/o adolescente, a la forma de posicionarse y adaptarse y a la secuencia de los hechos que acompañan vida del niño y/o adolescente en la apertura al plan terapéutico familiar.

El desarrollo de la etapa de intermedio de un plan terapéutico, se presentó de forma afirmativa en cuanto a la realización de la evaluación y el diagnóstico en pro de dar seguimiento a la apertura de los planes terapéutico familiares y en la formulación de hipótesis que contribuyan a la consulta y síntesis que el terapeuta debe hacer a partir de su formación teórica, para de esta forma aplicar técnicas variadas y de muchas fuentes en los niños y/o adolescente.

La etapa a final o etapa del cierre, de acuerdo a los investigados se debe dar luego de hacer una evaluación o un seguimiento de la problemática infanto-juvenil en el contexto familiar. Sin embargo, en menor porcentaje se señaló la intención, en algún momento por parte de las familias investigadas, abandonar el plan terapéutico. Además, se planteó la necesidad entre las familias investigadas, que una vez culminada la terapia familiar solicitar de nuevo una reunión de seguimiento. 


\section{Referencias Bibliográficas}

Bateson, G. (1999). Pasos hacia una ecología de la mente. Argentina .Lohlé-Lumen...

Edmond Gilliéron (s/f). Primeira Entrevista em Psicoterapia. Recuperado de: https://www.fnac.pt/A-Primeira-Entrevista-em-Psicoterapia-Edmond-Gillieron

Garaigordobil, M; Durá, A. y Pérez, J. (2005): Síntomas psicopatológicos, problemas de conducta y autoconcepto-autoestima: un estudio con adolescentes de 14 a 17 años. Anuario de Psicología Clínica y de la Salud, 1, 53-63.

Gimeno, A. (1999). La familia: el desafío de la diversidad. Barcelona. Ed. Ariel.

Gracia y Musitu (2000). Socialización familiar y valores en el adolescente: un análisis Recuperado de: https://www.raco.cat/index.php/AnuarioPsicologia/article/download/61542/88397

Guang, E. (2003). Avances en la práctica del proceso psicoterapéutico de la familia. Ecuador. CIF

Guay, F; Pantano, H y Boivin, M. (2003).Auto-concepto académico y logro académico: perspectivas del desarrollo sobre su ordenamiento causal. Diario de Psicología Educativa, $95,124-136$.

Navarro, E. (2006): Medida y relaciones de la autoestima en población psiquiátrica infantojuvenil. Valencia: Minim.

Salem, G. (1987). L'approche thérapeutique de la famille. París .Masson. 